\title{
Differentiation between Recurrent Glioma and Radiation Injury by Magnetic Resonance Spectroscopy Combined with Diffusion-Weighted Imaging
}

\author{
Wael M. Nemattalla', Laila M. El Kady', Tarek H. Khalil', Azza A. Gad', \\ Khaled E. Mohamed ${ }^{2} *$
}

Departments of ${ }^{1}$ Radiology and ${ }^{2}$ Neurosurgery, Suez Canal University Hospital, Ismailia, Egypt.

\begin{abstract}
:
Background: The aim of this study was to investigate the diagnostic efficiency of magnetic resonance (MR) spectroscopy with diffusion-weighted imaging in the evaluation of the recurrent contrast-enhancing regions at the location of treated gliomas. Patients and Methods: In 49 patients who had new contrast-enhancing lesions at the vicinity of previously resected and irradiated high-grade gliomas, single-voxel MR spectroscopy and diffusion-weighted imaging were performed. Spectral data for $\mathrm{N}$-acetylaspartate (NAA), creatine ( $\mathrm{Cr}$ ), choline (Cho), lipid (Lip), and lactate (Lac) were analyzed in combination with the apparent diffusion coefficient (ADC) in all patients. Diagnosis of these lesions was allocated by means of follow-up or histopathology. Results: The Cho/NAA and $\mathrm{Cho} / \mathrm{Cr}$ ratios were significantly higher in recurrent tumor group than in radiation injury group $(p<0.001)$. The ADC values and ADC ratios (quotient of ADC of contrastenhancing lesion and matching structure in the contralateral hemisphere) were significantly higher in radiation injury regions than in recurrent tumor $(p<0.001)$. With MR spectroscopy, two variables (Cho/NAA and $\mathrm{Cho} / \mathrm{Cr}$ ratios) were proved to differentiate recurrent glioma from radiation injury, and $81.5 \%$ of total patients were classified into correct groups. Using discriminant analysis for MR spectroscopy with diffusion-weighted imaging, three independent variables (Cho/NAA, Cho/Cr, and ADC ratio) could classify $91 \%$ of total patients into their correct groups. There was a significant difference between the diagnostic accuracy of the two discriminant analyses (Chi-square $=4.15, p=0.042$ ). Conclusion: MR spectroscopy combined with ADC ratio can enhance the ability to differentiate recurrent glioma from radiation injury.
\end{abstract}

Keywords: Magnetic resonance spectroscopy, Radiotherapy, Radiation injury, Tumor recurrence

\section{Introduction}

Gliomas constitute over $90 \%$ of primary brain tumors in persons older than 20 years $^{(1,2)}$. In many cases, the extent of surgical excision is limited by the involvement of neoplasm in vital or functional anatomic parts of the brain ${ }^{(3)}$. For this reason postsurgical external-beam radiother- apy is a generally accepted and common procedure for the management of gliomas $^{(4)}$. Enhancing lesions that arise on routine follow-up brain magnetic resonance imaging $(\mathrm{MRI})$ at the site of a previously identified and treated primary intracranial neoplasm may present a significant diagnostic dilemma. Many of these lesions do not have specific imaging characteristics that enable the radiologist to 
discriminant tumor recurrence from inflammatory or necrotic changes that result from treatment with radiation and/or chemotherapy ${ }^{(5)}$. MR spectroscopy and diffusion-weighted (DW) imaging are noninvasive functional imaging methods that provide information complementary to that of anatomic imaging, these methods have been proposed as alternative modalities for differentiating tumor recurrence and radia-tion ${ }^{(6,7)}$.

Single-voxel MR spectroscopy used in earlier investigations showed interpretative difficulties with overlapping metabolic ratios as a result of partial volume contamination $^{(8)}$. Multivoxel MR spectroscopy allows coverage of a larger volume and investigation of multiple regions of the lesion ${ }^{(7,9)}$. Nonetheless, multivoxel MR spectroscopy could not correctly classify lesions in about $18 \%$ of cases $^{(10,11)}$. Diffusion-weighted (DW) imaging has been considered a mean to characterize and differentiate morphologic features such as edema, necrosis, and tumor tissue by measuring differences in the apparent diffusion coefficient $(A D C)^{(12)}$. It was established that assessment of ADCs of enhanc-ing regions was useful in differentia-ting recurrent glioma and radiation injury ${ }^{(6,13)}$.

Both single-voxel MR spectroscopy and DW imaging could be carried out in our MR scanner. Both techniques were carried out on 49 patients as a try to differentiate recurrent glioma from radiation injury. The aim of this study was to investigate whether the addition of DW imaging parameters to MR spectroscopic data would improve the ability to differentiate the two groups.

\section{Patients and Methods:}

Forty-nine patients ( 27 male and 22 female; mean age, $35.77 \pm 16.88$ years, range 10-69 years) who had been subjected to surgical resection of brain tumors with pathology proven high-grade gliomas (histology according to the classification schemes of the World Health Organization: grade III, $n=34$; grade IV, $n=15$ ) were enrolled in the study. All patients had undergone a full course of conventional fractionated radiotherapy after resection, and all patients had developed new contrast-enhancing lesions at or near the site of the original treated tumor; these lesions were seen on planned follow-up MR images. Written informed consents were obtained from all patients after explaining the background of the examinations. The Medical Ethical Committee of Suez Canal University, Faculty of Medicine, approved the study.

Follow-up MR Scans were performed 6 weeks after completion of radiotherapy and at 3-4 month intervals, depending on the clinical course. MR spectroscopy and DW imaging examinations were performed after initial identification of the recurrent contrast-enhancing lesion on follow-up MR images.

Histopathologic examination and followup MR imaging (after MR spectro-scopy and diffusion-weighted imaging) were used to ascertain the characteristics of the contrast-enhancing lesion. Lesions were considered tumor recurrence if they showed later histopathologic evidence of tumor (by biopsy or surgical resection) or if they showed mass effect and steady increase of enhancement at the follow-up MR images. Lesions were classified as radiation injury if they showed later histopathologic confirmation of radiation injury (by biopsy or surgical resection) or if they showed stable or resolving areas of contrast-enhancement on subsequent $M R$ image.

Tissue specimens were obtained from 16 patients by biopsy, 5 by means of stereotactic biopsy, and 11 by means of surgi- 
cal resection; biopsies were taken by neurosurgeons. Of the specimens, 12 were classified as tumor recurrence and 4 as radiation injury. The remaining 33 patients underwent additional conventional MR follow-up after the initial functional imaging; the follow-up time was 6.36 months (range, 3-12) where 8 patients were classified as tumor recurrence and 25 patients were classified as radiation injury.

\section{$M R$ and DW imaging}

All examinations were performed on a 1.5T MRI system (Achieva, Philips Medical Systems, Best, The Netherlands). The conventional MR images consisted of axial T1weighted (500/11 ms [TR/ TE]) spin-echo (SE), T2-weighted (4000/100 ms) fast SE, and fluid-attenuated inversion-recovery (FLAIR) (9000/120/ $2250 \mathrm{~ms}$ [TR/TE/TI]) images obtained by using 6-mm section thickness, 240-mm field of view (FOV), and $320 \times 224$ matrix.

The DW images were obtained by using an axial echo-planar SE sequence (5000/65 ms [TR/TE]; one average; 6-mm thickness; diffusion gradient encoding in three orthogonal directions; $b=100 \mathrm{~s} / \mathrm{mm}^{2}$; 240-mm FOV; $160 \times 192$ matrix) in $1 \mathrm{~min}$. Contrast-enhanced T1-weighted SE images were then obtained in axial, coronal, and sagittal planes after intravenous administration of gadopen-tetate dimeglumine (Magnevist, Schering, Berlin, Germany) at a dose of $0.1 \mathrm{mmol} / \mathrm{kg}$ of body weight. Post processing of ADC maps was performed by using dedicated standard software on a workstation. Regions of interest (ROIs) were drawn manually onto the obtained ADC maps in the regions matching to the enhancing areas on contrastenhanced T1-weighted images. The dedicated software calculated the $A D C$ value automatically. The ADC ratio (normalized $A D C$ value) was calculated as the quotient of the $A D C$ value of the enhancing region and the matching structure of same-size $\mathrm{ROI}$ in the contralateral hemisphere.

\section{MR spectroscopy}

MR spectroscopy was performed after 1 hour from contrast-enhanced MR imaging to minimize the effect of gadolinium on MR spectroscopy. The following parameters were used for MR spectroscopy: a point-resolved spectroscopy sequence (PRESS), which included water and outer volume saturation pulses; 1500/144 ms [TR/TE]; 16-cm FOV; $16 \times 16$ matrix; 10-mm slice thickness; 1 average acquisition; scanning time, $4 \mathrm{~min}$. The volume of interest (VOI) was placed on axial T1-weighted images matching to the contrastenhancing area on contrast-enhanced axial T1-weighted mages. Automatic prescanning was performed before each spectroscopic scan to guarantee adequate water suppression. The full-width half-maxi-mum was kept at less than $15 \mathrm{~Hz}$ and water saturation between 95-99\%.

Within the obtained VOI, separate $1 \times 1 \times 1 \mathrm{~cm}$ voxels were positioned in the area of enhancement. Metabolite peaks used were as follows: $\mathrm{N}$ - acetylaspartate (NAA) at 2.02-ppm, choline-containing compounds (Cho) at 3.22-ppm, (phospho-) creatine $(\mathrm{Cr})$ at 3.02-ppm, lipid-containing compounds (Lip) in the range of 0.9-1.3 ppm, and lactate (Lac) at 1.33-ppm (inverted $\beta$-methyl doublet). Metabolite values were calculated automatically from the area under each metabolite peak by the dedicated software. The metabolite ratios (NAA/Cr, Cho/Cr, $\mathrm{Lip} / \mathrm{Cr}$ and $\mathrm{Lac} / \mathrm{Cr}$ ) were calculated by the software and the Cho/NAA was manually calculated.

\section{Statistical analysis}

Statistical analysis was performed using SPSS for Windows software, release 11.5 (SPSS Inc., Chicago, IL). Metabolite ratios and $A D C$ parameters (ADC value and ADC 
ratio) between the recurrent tumor group $(n=20)$ and radiation injury group $(n=29)$ were compared using the unpaired, twotailed Student $\boldsymbol{t}$ test. A forward stepwise discriminant analysis was carried out to evaluate the power of metabolite ratios and ADC parameters to distinguish tumor recurrence and radiation injury. Diagnostic accuracy was then compared between metabolite ratios alone and metabolite ratios besides ADC parameters for discriminating the two entities by using the Chi-square test. The level of significance was set at $p<0.05$.

\section{Results}

Findings of MR spectroscopy

The $\mathrm{Cho} / \mathrm{Cr}$ and Cho/NAA ratios of the contrast-enhancing lesions in the recurrent tumor group were significantly higher than those in the radiation injury group $(\mathrm{p}=0.001$ and $\mathrm{p}=0.001$ respectively), whereas the $\mathrm{NAA} / \mathrm{Cr}$, $\mathrm{Lac} / \mathrm{Cr}$ and $\mathrm{Lip} / \mathrm{Cr}$ ra- tios of the contrast-enhancing lesions in the recurrent tumor group were significantly lower than those in the radiation injury group $(p=0.001, p=0.001$ and $p=0.001$ respectively). The mean values of the metabolite ratios in relevant lesions are shown in Table 1. Representative MR images, MR spectroscopic images and diffusion-weighted ADC map images are shown in Figures 1 and 2.

\section{Findings of DW imaging}

The recurrent tumor group showed significantly lower $A D C$ value $\left(1.12 \pm 0.14 \mathrm{~mm}^{2} / \mathrm{s}\right.$, mean $\pm S D$ ) compared with the radiation injury group $\left(1.44 \pm 0.11 \mathrm{~mm}^{2} / \mathrm{s}\right) ; p=0.001$. The normalized ADC (ADC ratio) was significantly lower in the recurrent tumor group (1.40 \pm 0.08$)$ compared to that in the radiation injury group (1.68 \pm 0.1$) ; p=0.001$. $A$ box-and-whisker diagram of the $A D C$ values and $A D C$ ratios was used to illustrate these results in Figures $3,4$.

Table 1: Calculated magnetic resonance spectroscopy ratios in various groups

\begin{tabular}{|l|lllll|}
\hline Groups & Cho/Cr & Cho/NAA & Lac/Cr & Lip/Cr & NAA/Cr \\
\hline Tumor recurrence $(n=20)$ & $2.7 \pm 0.68$ & $3.07 \pm 0.71$ & $1.04 \pm 0.16$ & $0.31 \pm 0.10$ & $0.87 \pm 0.23$ \\
Radiation injury $(n=29)$ & $1.81 \pm 0.64$ & $1.78 \pm 0.63$ & $1.81 \pm 0.64$ & $0.64 \pm 0.18$ & $1.31 \pm 0.31$ \\
\hline
\end{tabular}

Abbreviations: Cho=choline-containing compounds; $\mathrm{Cr}=$ (phospho-)creatine; Lac=lactate; Lip= lipidcontaining compounds; $\mathrm{NAA}=\mathrm{N}$-acetyl-aspartate. Data are presented as Means $\pm \mathrm{SD}$

Findings of MR spectroscopy combined with DW imaging

To evaluate the power of correct classification, two discriminant analyses were carried out for metabolite ratios alone and for metabolite ratios combined with ADC parameters. In the first analysis, $\mathrm{NAA} / \mathrm{Cr}$, Cho/Cr, Lip/Cr, Lac/Cr, and Cho/NAA were used as independent variables. In the second analysis, all variables (metabolite ratios and ADC parameters) were used as independent variables. For both analyses, the findings of follow-up or histopathology provided the group variable. In the first analysis, two variables were significant. The Cho/NAA value appeared as the first variable to differentiate tumor recurrence from radiation injury. To settle the ability of other variables, Cho/ NAA was removed and $\mathrm{Cho} / \mathrm{Cr}$ came out as the second variable. When $\mathrm{Cho} / \mathrm{Cr}$ was expelled, other variables did not contribute significantly. 
When both variables (Cho/NAA and $\mathrm{Cho} / \mathrm{Cr}$ ) were assembled together for classification, $81.5 \%$ of total subjects were classified correctly according to radiation injury (83\%) versus tumor recurrence (80\%) (Table 2).

In the second analysis, three significant variables were identified. The ADC value did not add significantly to differentiate tumor recurrence from radiation injury. When all the three variables $(\mathrm{Cho} / \mathrm{Cr}$, Cho/NAA, and ADC ratio) were introduced together for classification, $91 \%$ of total subjects were classified into correct groups (radiation injury, 97\%; tumor recurrence, $85 \%$ ) Compared with the first analysis, the second analysis could increase the accuracy of discriminant analysis for the assessment of the recurrent enhancing lesion (Chi-square $=4.15, p=0.042$ ). On the basis of discriminant analysis, two different equations were derived (Table 2). If $D$ (tumor recurrence) was more than $\mathrm{D}$ (radiation injury), the lesion was classified as tumor recurrence. If $D$ (radiation injury) was more than $\mathrm{D}$ (tumor recurrence), the lesion was classified as radiation injury.

Table 2: Results of stepwise discriminant analyses (SDA)

\begin{tabular}{|l|lcccc|}
\hline & Variable & Tumor recurrence & Radiation injury & Group & Classified (\%) \\
\hline $\begin{array}{l}\text { SDA for me- } \\
\text { tabolite ratios }\end{array}$ & Cho/NAA & 2.87 & 0.72 & Tumor recurrence & 80 \\
& Cho/Cr & 7.25 & 5.12 & Radiation injury & 83 \\
\hline $\begin{array}{l}\text { SDA for com- } \\
\text { bined metabo- }\end{array}$ & Cho/NAA & 6.55 & -5.43 & Total & 81.5 \\
$\begin{array}{l}\text { lite ratios and } \\
\begin{array}{l}\text { ADC parame- } \\
\text { ters }\end{array}\end{array}$ & Cho/Cr & 8.16 & 5.23 & Tumor recurrence & 85 \\
& ADC ratio & 176.55 & 6.30 & Radiation injury & 97 \\
\end{tabular}

Abbreviations: ADC ratio=quotient of apparent diffusion coefficient of contrast-enhancing lesion and matching structure in contralateral hemisphere; Cho=choline-containing compounds; $\mathrm{Cr}=($ phospho-) creatine; Lac=lactate; Lip=lipidcontaining compounds; $\mathrm{NAA}=\mathrm{N}$-acetyl-aspartate. Based on the discriminant analysis, the discriminant equation is: For metabolite ratios: $\mathrm{D}$ (tumor recurrence) $=2.87(\mathrm{Cho} / \mathrm{NAA})+7.25(\mathrm{Cho} / \mathrm{Cr})-17.04, \mathrm{D}$ (radiation injury) $=0.72(\mathrm{Cho} / \mathrm{NAA})$ $+5.12(\mathrm{Cho} / \mathrm{Cr})-5.43$. For combined metabolite ratios and $A D C$ parameters: $\mathrm{D}$ (tumor recurrence) $=6.55(\mathrm{Cho} / \mathrm{NAA})+$ $8.16(\mathrm{Cho} / \mathrm{Cr})+176.55(\mathrm{ADC}$ ratio $)-149.68, \mathrm{D}$ (radiation injury $)=5.23(\mathrm{Cho} / \mathrm{NAA})+6.30(\mathrm{Cho} / \mathrm{Cr})+198.39(\mathrm{ADC}$ ratio $)-$ 181.05

\section{Discussion}

When encountering an indeterminate $\mathrm{T} 1$ enhancing lesion after radiation therapy for a high-grade tumor then we have common diagnostic dilemma; what is that focal lesion, "radiation injury", or "recurrent tumor"? Recurrent glioma and radiation inju ry are difficult to distinguish due to shared MR imaging characteristics such as areas of abnormal enhancement with surrounding edema $^{(14-16)}$. The conventional MR imaging findings as clues to diagnose recurrent tumor and radiation injury have been disput$\mathrm{ed}^{(14)}$. 

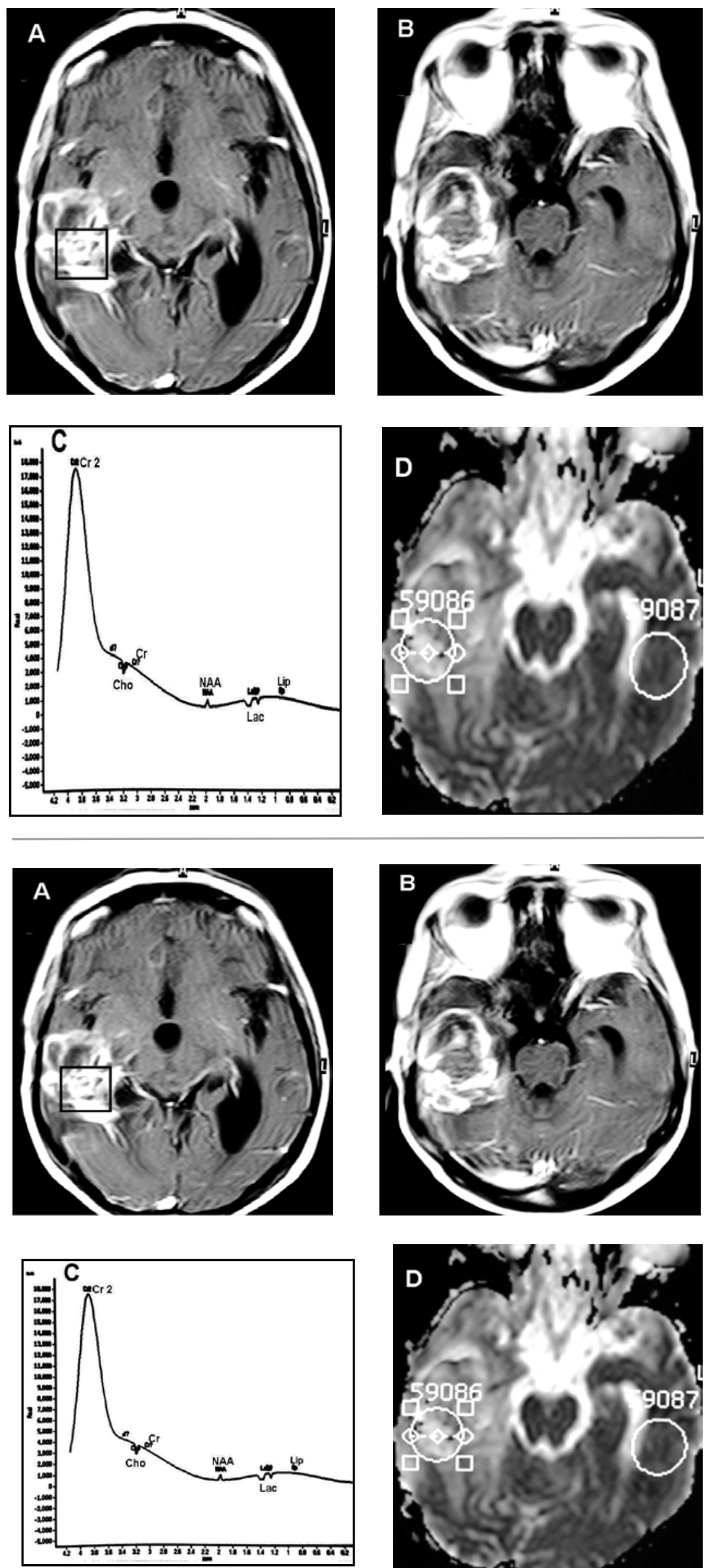

Figure 1 Radiation necrosis in a 58-year-old female who underwent surgery and radiotherapy for right temporal lobe anaplastic astrocytoma. (A) and (B) Contrast-enhanced T1-weighted axial images demonstrate large complex enhanced area with central necrosis. Volume of interest of magnetic resonance (MR) spectroscopy is shown in (A). (C) MR spectroscopic image shows markedly diminished metabolites except Lip and $\mathrm{Cr} 2$. Area under curve for Lip (0.9-1.3 ppm) is increased. (D) Apparent diffusion coefficient (ADC) map image $(b=$ $1000 \mathrm{~s} / \mathrm{mm}^{2}$ ) shows no evidences of diffusion restriction with $\mathrm{ROI}$ is placed onto solid contrastenhancing part of the lesion. Mean ADC value was $1.76 \times 10^{-3}$ $\mathrm{mm}^{2} / \mathrm{s}$ and $\mathrm{ADC}$ ratio was 1.88 . Biopsy of this lesion revealed radiation necrosis.
Figure 2: Tumor recurrence in a 69year-old male who underwent surgery and radiotherapy for left parietal glioblastoma multiforme (A) \& (B) Contrast-enhanced T1-weighted axial images demonstr-ate large enhanced area with irregular walls surrounded by edema with no midline shift. (C) MR spectroscopic image shows markedly increased Cho with increased $\mathrm{Cho} / \mathrm{Cr}$ and Cho/NAA ratios (3.74 and 4.1 respectively). Small increase in Lac is also seen with $\mathrm{Lac} / \mathrm{Cr}$ ratio $=0.6$. Apparent diffusion coefficient (ADC) map image $\left(b=1000 \mathrm{~s} / \mathrm{mm}^{2}\right)$ with ROI placed onto solid contrast-enhan-cing part of the lesion. Some diffusion restriction is seen as dark area within ROI. Statistics for ROls showed mean ADC value in the contrast-enhancing lesion of $1.15 \times 10^{-3} \mathrm{~mm}^{2} / \mathrm{s}$ and ADC ratio of 1.36. Biopsy of this lesion revealed hypercellular glioblastoma multiforme with areas of radiation changes. 
In an attempt to overcome this problem; we applied in our study a combination of two MR imaging modalities (MR spectroscopy and diffusion-weighted imaging) aiming to increase the accuracy of differentiation between these entities. Either MR spectroscopy or DW imaging has been used to distinguish tumor recurrence from radiation injury. These physiology based imaging methods (MRS and DWI) can direct neurosurgeon and pathologist to obtain biopsy from the appropriate sites and to provide appropriate histopathologic evaluation. Furthermore, any sequential therapeutic measures depend on the correct classification ${ }^{(17,18)}$.
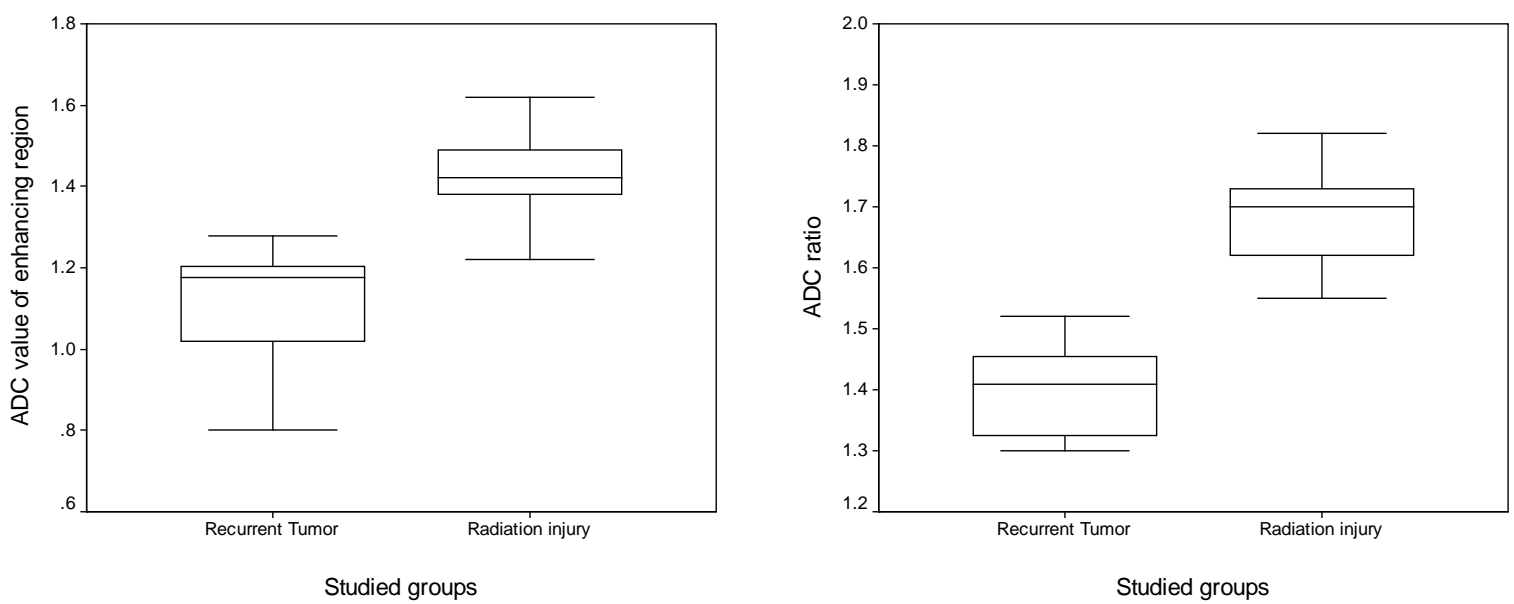

Figure 3: Comparison of apparent diffusion coefficient values $\left(\times 10^{-3} \mathrm{~mm}^{2} / \mathrm{s}\right)$ between the recurrent tumor and radiation injury groups. Line indicates the range of data; boxes represent the distance between the first and third quartiles, with the median between them. Figure 4 Comparison of apparent diffusion coefficient ratio between the recurrent tumor and radiation injury groups. Line indicates the range of data; boxes represent the distance between the first and third quartiles, with the median between them.

The main findings of the current study were: (1) Significant differences in the studied metabolite ratios $(\mathrm{Cho} / \mathrm{Cr}$, Cho/NAA, NAA/Cr, $\mathrm{Lac} / \mathrm{Cr}$ and $\mathrm{Lip} / \mathrm{Cr}$ ) and $A D C$ parameters (values and ratios) were found between the tumor recurrence and radiation injury groups. (2) With discriminant analysis, $\mathrm{Cho} / \mathrm{Cr}$, Cho/NAA, and ADC ratios were the main factors to distinguish recurrent tumor from radiation injury, and the addition of ADC ratios to MR spectroscopy ratios could increase the power of differentiation for the two entities. In our stepwise discriminant analysis, the first classification vector was correlated highly with Cho/NAA. The second classification vector concerned mainly $\mathrm{Cho} / \mathrm{Cr}$.
According to the classification vectors we used in our study to differentiate between the two groups (radiation injury and tumor recurrence); 40 patients (81.5\%) were classified into correct groups; $80 \%$ of recurrent tumor patients could be correctly classified (16 out of 20 patients); $83 \%$ of radiation injury patients could be correctly classified (24 out of 29 patients). These results are in close agreement with the previous studies $^{(10,11) \cdot}$ In the study of Lichy et al $^{(11)}$ using 2D MR spectroscopy, Cho/Cr and Cho/NAA ratios allowed a correct classification in more than $80 \%$ of the cases which is in excellent match with our results. A comparable result was found in the study of Zeng et al $^{(19)}$ who got an ac- 
curacy of $85.5 \%$ for the combined $\mathrm{Cho} / \mathrm{Cr}$ and Cho/NAA ratios in differentiating tumor recurrence from radiation injury. Matching of our results with that of Zeng might be explained on basis of near similarity in both the sample size (49 patients in our study versus 55 patients in his study) and the statistical methods used.

The apparent diffusion coefficient (ADC) values of biologic tissue reflect the Brownian movement of water molecules, which reveal potentially histopathologic characteristics of cellular structure ${ }^{(13)}$. Cellularity, is an important factor that influences ADC in brain tumors (after therapy). Previously, higher cellularity in recurrent neoplasm would contribute to lower ADC values $^{(20)}$. Significant differences in ADC value were found between the two groups in our study, which is explained by the fact that recurrent growth of tumor determines to great extent the tissue ADC.

In our study; the function of discriminant analysis was interrelated highly with combined $\mathrm{Cho} / \mathrm{Cr}$, Cho/NAA and $\mathrm{ADC}$ ratio in the second analysis; the ADC value alone did not add through discriminant analysis to further discriminant tumor recurrence and radiation injury; this result is in excellent agreement with previous studies $^{(19,21)}$. In the study by Rock et al. ${ }^{(21)}$ on 18 patients with malignant glioma previously treated with surgery and radiotherapy; the authors concluded that metabolite ratio analysis might allow for discrimination between specimens of tumor and necrosis. However, the direct addition of ADC value to MR spectroscopy did not add to the value of MR spectroscopy in distinguishing the two groups. In our study, a highly significant difference of the $A D C$ ratio between the two entities was observed. Additionally, DW imaging could assess entire contrast-enhancing regions ${ }^{(22)}$ which may defeat the shortcom- ing of 2D MR spectroscopy that only confines a limited region size. For these reasons, when the three variables $(\mathrm{Cho} / \mathrm{Cr}$, Cho/NAA, and ADC ratio) were concerned together for classification in the second discriminant analysis, $91 \%$ of the total patients were correctly-classified.

Biopsy is the only 'gold standard' reference test and is desired in all patients. Due to absence of histopathological verification of the final diagnosis; most of the current imaging modalities gave a low level of evidences when reviewing their accuracy in differentiating tumor recurrence from radiation necrosis ${ }^{(23)}$. Biopsy was not available in all of our patients; to overcome this problem clinical and imaging follow-up in our patients were an alternative which is accepted by Alexiou et al. ${ }^{(23)}$. In our study; histopathologic confirmation was in $32.6 \%$ of patients and follow-up imaging was applied for $67.4 \%$ of patients. McGirt et $\mathrm{al}^{(24)}$ reported that histopathologic confirmation is not always clinically practicable because of a high risk of morbidity and approximately $10 \%$ sampling error in biopsy cases ${ }^{(24)}$. Small tumor cell clusters may be missed by biopsy specimens, which are not necessarily representative for the whole, heterogeneous lesion $^{(10)}$. Another limitation in our study is the short follow-up of these lesions after MR spectroscopy (6.36 months; range, 312 months). A prolonged follow-up imaging of contrast-enhancing lesions after MRS is desirable in all cases to minimize the possibility of misclassification. Additional limitation in our study is the variability in the timing of follow-up imaging, which is influenced by the distinctive clinical courses of the different patients.

Conclusion Our results suggest that $M R$ spectroscopy allows a noninvasive differentiation of recurrent gliomas from radiation injury in patients with indeterminate 
findings on routine follow-up MR images. Adding the ADC ratio to MR spectroscopy signify-cantly improves the ability to differentiate the two entities. The accuracy of differ-rentiation of MR spectroscopy in combination with $A D C$ ratio is higher than that of MR spectroscopy alone.

\section{References}

1. Kleihues $P$, Soylemezoglu F, Schéauble B, Scheithauer BW, Burger PC. Histopathology, classification, and grading of gliomas. Glia. 1995; 15:211-221.

2. Lantos PL, Vandenburg SR, Kleihues P. Tumours of the nervous system. In: Graham D, Lantos P, eds. Greenfield's Neuropathology (6th ed.). New York, NY: Oxford University Press 1997:583-593.

3. Kreth FW, Berlis A, Spiropoulou V, et al. The role of tumor resection in the treatment of glioblastoma multiforme in adults. Cancer 1999; 86:2117-2123.

4. Herfarth KK, Gutwein S, Debus J. Postoperative radiotherapy of astrocytomas. Semin Surg Oncol 2001; 20:13-23.

5. Bonavita S, Di Salle F and Tedeschi G. Proton MRS in neurological disorders. Eur Radiol 1999; 30:125-131.

6. Asao C, Korogi Y, Kitajima M, et al. Diffusion-weighted imaging of radiationinduced brain injury for differentiation from tumor recurrence. Am J Neuroradiol 2005; 26:1455-1460.

7. Weybright $P$, Sundgren PC, Maly P, et al. Differentiation between brain tumor recurrence and radiation injury using $M R$ spectroscopy. AJR Am J Roentgenol 2005; 185 (6):1471-1476.

8. Schlemmer JP, Bachert P, Henze M, et al. Differentiation of radiation necrosis from tumor progression using proton magnetic resonance spectroscopy. Neuroradiology 2002; 44: 216-222.

9. Rabinov JD, Lee PL, Barker FG, et al. In vivo 3-T MR spectroscopy in the distinction of recurrent glioma versus radiation effects: Initial experience. Radiology 2002; 225:871-879.
10. Schlemmer HP, Bachert P, Herfarth K, et al. Proton MR spectroscopic evaluation of suspicious brain lesions after stereotactic radiotherapy. Am J Neuroradiol 2001; 22:1316-1324.

11. Lichy MP, Plathow C, Schulz-Ertner D, et al. Follow-up gliomas after radiotherapy: $1 \mathrm{H}$ MR spectroscopic imaging for increasing diagnostic accuracy. Neuro-radiology 2005; 47:826-834.

12. Castillo M, Smith JK, Kwock L, Wilber K. Apparent diffusion coefficients in the evaluation of high-grade cerebral gliomas. Am J Neuroradiol 2001; 22:60-64.

13. Hein PA, Eskey CJ, Dunn JF, Hug EB. Diffusion-weighted imaging in the follow-up of treated high-grade gliomas: tumor recurrence versus radiation injury. Am. J. Neuroradiol 2004; 25: 201-209.

14. Mullins ME, Barest GD, Schaefer PW, et al. Radiation necrosis versus glioma recurrence: Conventional MR imaging clues to diagnosis. Am J Neuroradiol 2005;26:1967-1972.

15. Law M, Young RJ, Babb JS, Peccerelli N, Chheang S, Gruber ML, Miller DC, Golfinos JG, Zagzag D, Johnson G. Gliomas: Predicting Time to Progression or Survival with Cerebral Blood Volume Measurements at Dynamic Susceptibilityweighted Contrast- enhanced Perfusion MR Imaging. Radiology 2008; 247: 490498.

16. Cha S. Neuroimaging in neuro-oncology. Neurotherapeutics, 2009; 6 (3):465-477.

17. Kallen K, Burtscher IM, Holtas S, et al. 201 Thallium SPECT and $1 \mathrm{H}-\mathrm{MRS}$ compared with MRI in chemotherapy monitoring of high-grade malignant astrocytomas. J Neurooncol 2000;46:173-185.

18. Kocaoglua $M$, Ors $F$, Bulakbasi $N$, Ongurub O, Ulutinc, C, Secer HI. Central neurocytoma: proton MR spectroscopy and diffusion weighted MR imaging findings. Magn Reson Imaging 2009; 27(3):434-440.

19. Zeng $\mathrm{QS}$, Li CF, Liu H, Zhen JH. Feng DC. Distinction between recurrent glioma and radiation injury using magnetic resonance spectroscopy in combination with diffusion-weighted imaging. Int. J. Radia- 
tion Oncology Biol. Phys. 2007; 68 (1): 151158.

20. Kono K, Inoue $\mathrm{Y}$, Nakayama K, et al. The role of diffusion-weighted imaging in patients with brain tumors. AJNR Am J Neuroradiol 2001; 22:1081-1088.

21. Rock JP, Scarpace L, Hearshen D, et al. Associations among magnetic resonance spectroscopy, apparent diffusion coefficients and image-guided histopathology with special attention to radiation necrosis. Neurosurgery 2004;54 (5): 1111-1119.

22. Bammer R. Basic principles of diffusionweighted imaging. Eur J Radiol 2003; 45:169-184.

23. Alexiou GA, Tsiouris $S$, Kyritsis AP, Voulgaris S, Argyropoulou MI, Fotopoulos AD. Glioma recurrence versus radiation necrosis: accuracy of current imaging modalities. J Neurooncol. 2009; 95 (1): 1-11.

24. McGirt MJ, Woodworth GF, Coon AL, et al. Independent predictors of morbidity after image-guided stereotactic brain biopsy: A risk assessment of 270 cases. J Neurosurg 2005; 102:897-901. 